\title{
The pervasive nature of DAM across a multimedia organization
}

\author{
Neil McLaren \\ is one of the co-founders of the Arkemedia software and has been working in digital asset management (DAM) since its early days \\ seven years ago. A marketing specialist, his background encompasses media and IT, as he spent time before developing Arkemedia \\ working with a Digital Equipment Corporation in technical marketing.
}

Keywords: multimedia, broadcasters, analog, internet, repurposed, XML, advertising

\begin{abstract}
For most businesses operating in the media sector, a digital asset management (DAM) solution is, fundamentally, an electronic cataloguing tool which allows a wide group of consumers to search, store and utilize content through copying and distribution of the content itself and its associated metadata. In many cases, workflow controls monitor the status of content or metadata. Enterprises, however, need to apply a high number of business rules to determine content usage - for example, a 30-second commercial required for broadcast. On its own, the rules associated with a commercial are limited, but the business rules operating on this content ring from ownership of an advertiser, agency and internal sales executive through to a booking mechanism, invoicing and associated financial policy. These rules have traditionally been part of a sales or traffic system and simply act on data or data types. In this sense each set of data is an object. With content stored as an object, the logical development is a DAM solution. A DAM solution considers these business rules, allows them to act upon the content and therefore become a pervasive application and primary business support tool across any media player. This paper attempts to argue the case that this is a logical conclusion to the introduction and development of DAM systems.
\end{abstract}

\section{THE ANALOG WORLD OF BROADCASTERS}

For anybody who has worked in the broadcast and media industry for the past two decades, it feels very much like we have lived through a communications revolution. A snapshot view of the world 20 years ago reveals there were few media outlets and high barriers to entry for new players. Each media player was often limited, through regulation or pure investment, to one vertical market.

In its analog state, publishable and transmittable material existed in many forms (eg $35 \mathrm{~mm}$ film, $2^{\prime \prime}$ tapes, photo negatives hard copy scripts). Over time, there has been a steady migration from paper-based control and publishing mechanisms to computerized

Neil McLaren Harris Corporation. View Point, Basing View, Basingstoke, Hampshire, RG21 4RG UK

Tel: +441256869200 Email: nmclaren@harris.com management of data - common to much business across the corporate landscape.

In the broadcast world, paper contracts and paper schedules for example, have now become computerized data items, with multiple properties and behavior characteristics. The major difference today is that written conditions can be encapsulated as a set of rules to govern the usage of the data item and therefore the content associated with it. It is important here to note that rules could be created rather than imposed from interpretation of a contract or agreed business activity.

\section{THE ADVENT OF DIGITIZATION}

There can be no doubt that the advent of digitization has changed and will continue to change the media industry dramatically. Arguably, it has in the process, created the need for digital asset management (DAM) solutions.

Digitization has increased the distribution of content $n$-fold, lowered the barriers to entry and forced legislators to review ownership rules and how media are regulated. At a purely technical level, the conversion of content to a commondenominator form has enabled the merger of previously separated businesses into multimedia outlets. In addition, it has forced vendors to use 


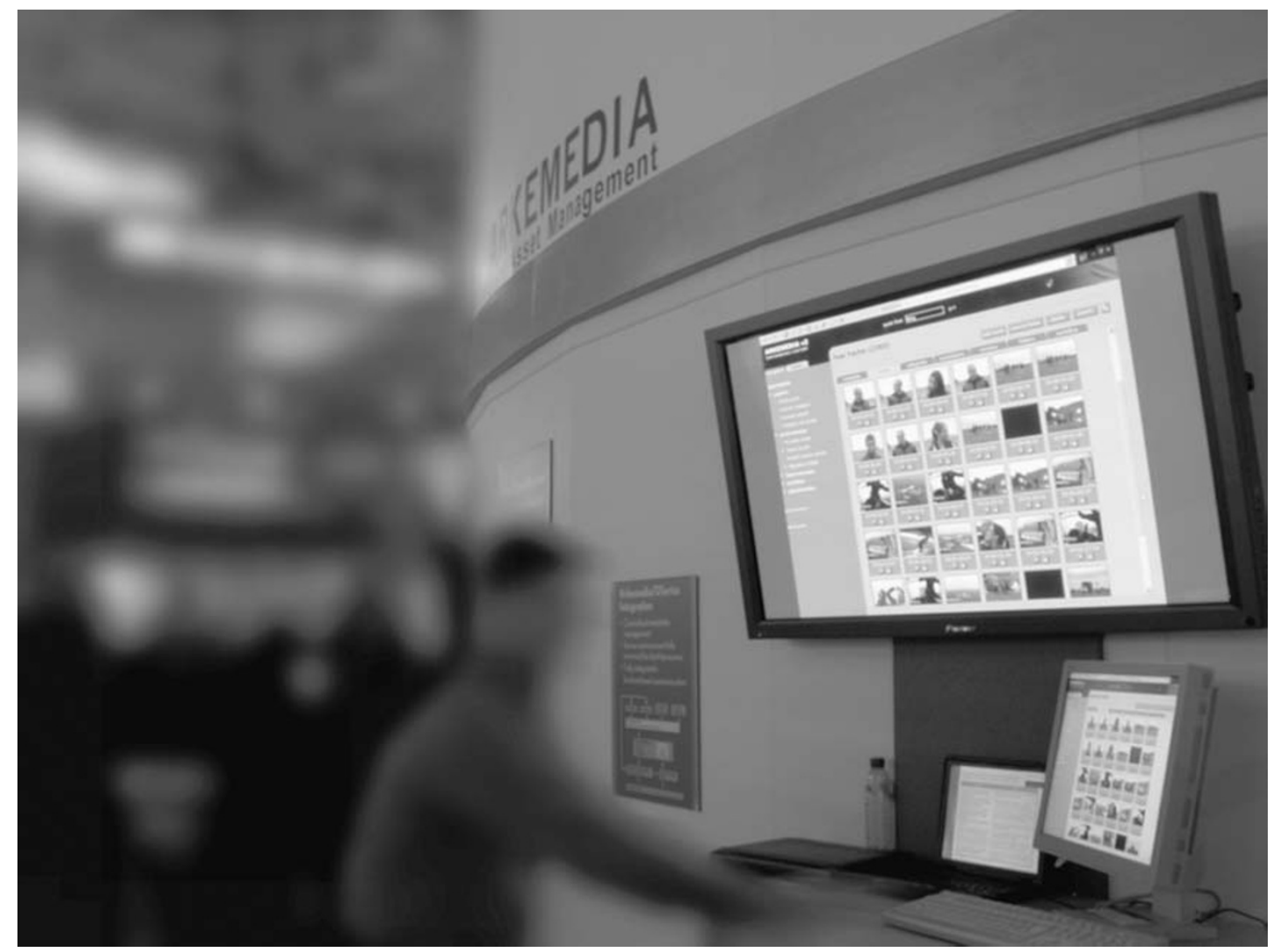

Figure 1: Arkemedia demo as shown at IBC 2004: The Arkemedia storyboard appears onscreen

common technology platforms or at least operate in an open environment to allow for the full use of digitized content across business and multiple applications.

Repurposing of this content is now the key to lowering costs for a multimedia operator, with the technology solution being a content or DAM system. The growth of the number of vendors delivering this type of solution and the number of media players actively engaged in the sales cycle is testament to this.

On their own, the benefit DAM systems can bring is not fully realized. However, setting the business rules on top of DAM offers the holy grail of a true enterprise-wide business application support based on content.

\section{THE ROLE OF DAM WITHIN A MEDIA OPERATION}

DAM focuses on the workflow of content preparation and can allocate arbitrary values, but does not apply a complex set of business rules governing allowance and behavior characteristics.

The role of DAM within media operations varies greatly depending upon who you ask. Some have considered DAM's role and have developed or implemented it according to a linear set of needs that makes content easier to find or enables its transfer from one system to another. As with all new technology, the way it is embraced and utilized is invariably less than its total potential. This goes some way to explaining the experience of first-to-market suppliers and early-adopting users.

However, those who have watched this new technology grow from its embryonic state and have anticipated the wider relevance of DAM paint an entirely different picture. The role of DAM can, when applied to its full potential, provide an all-embracing overarching layer of software over an operation that concerns itself with every element of the media workflow.

The reliance on legacy systems and extracting 


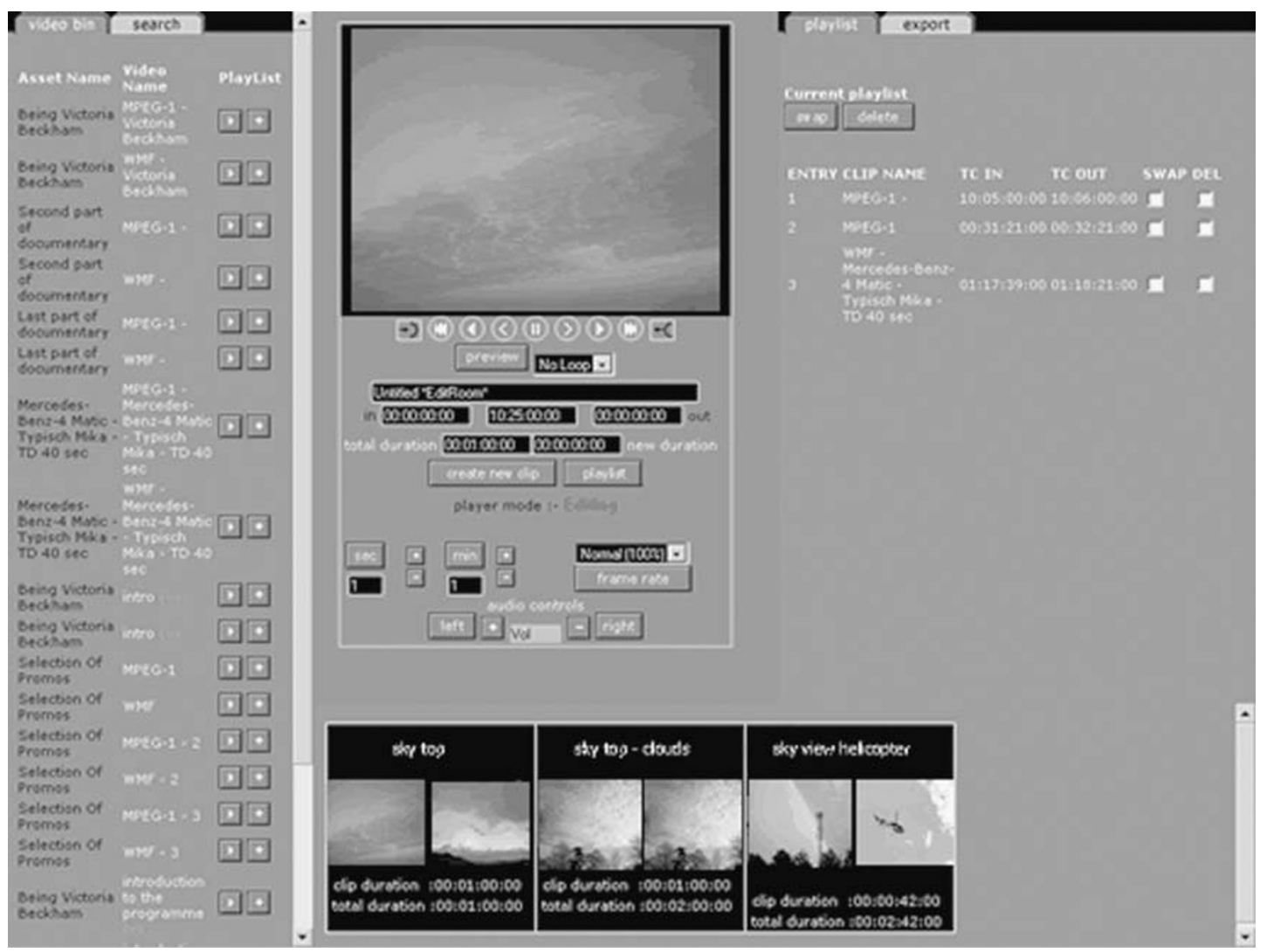

Figure 2: An Arkemedia screenshot of Edit Room, a unique feature in DAM

the full benefit of previous investments (media companies are now as business savvy as the next corporate) means that extensive overhaul and replacement of systems are not options. Media operations demand more return from their existing set-ups and this is where DAM comes in to its own, when written and implemented well.

DAM can help to extract the full potential of systems and processes that were established prior to its own deployment and in considering this, we near the crux of what its role is to some and should be to others.

There are tools within most DAM offerings which catalog, digitize and make content easier to find. Functionality may also be offered that allows some processes that have traditionally taken place within dedicated systems to occur within the DAM environment. However, where good DAM really holds its own is when this layer of software looks into and collects data from all technological islands, which have grown within facilities and media processes, and makes them smoother and more efficient.

Media operations are fluid and often chaotic environments, so applying systems that provide a single view into any constituent element of the workflow means that not only can content be found and tracked, but it is auditable along with the systems with which it is integrated and the users who work with the content.

For some people, developing a myopic view where attention is only paid to the tools "within" the DAM system ("my search tools must be faster") is an unfortunate given. Yet it precludes the benefits offered when taking a wider view of what such technology can do to improve efficiency and thus, the bottom line.

\section{REVIEW OF THE PRACTICES OF A MEDIA ORGANIZATION}

If one examines the business flow within a media organization it is primarily based around content 
acquisition, leveraging that content value through commercial activity and distributing it to the consumer. Within this broad outline, many business models exist.

Regardless of the model, however, business rules are operating on the content in some form. Below are outlined some of these business rules which are associated to content, enough, it is hoped, to give the reader an idea of a fuller utilization of DAM systems.

\section{BUSINESS RULES}

Generally, no media player will produce all the content that it distributes. In most cases, producers create content and sell the rights to a third party. Likewise, broadcasters engage in acquisition of some form, which gives use of content for a period of time based on a contractual agreement. This arrangement, digitized as a set of rules within a business system will, for example, control usage, distribution channel, rights windows and amortization policy. There is not just a value allocated to the content, but a cost of usage.

The scheduling component of the media management system will observe the rights' details and ostensibly ensure that any scheduling of content conforms to the contractual arrangement. This is of course subject to the data being entered correctly by users of the system.

Currently, there is very little verification by a rights seller that the terms of a contract have been honored in their entirety by the purchaser. This of course may change over the next few years with the introduction of rights encoding standards, such as MPEG-21. In fact, it is possible that encoding rights with content may be the catalyst for allowing business rules to act on content through DAM systems.

Generating commercial value from the content will also require a business set of rules. In essence, the business rules are broken down into two key components: first business intelligence data, which enable an organization to maximize revenue from its assets and distribution channels and secondly, sales workflow - that functionality which manages the sales process from initiation to revenue receipt.

In a broadcast environment, business intelligence data can prove complex, as targeting individuals is not possible, this being broadcast rather than narrowcast. Complex models of the audience behavior are used to determine which demographic should be associated with specific event or content transmission at a specific date or time.

Although these models are developed by specific research organizations, with the media player a third-party user of the information, this key information needs to be held against the content in such a way as to allow the media operation to maximize the value of this content. Complex algorithms are used to highlight best performing demographics for the specific content event within the context of a schedule for a particular period of time, whether it be day, week or month.

In a traditional broadcast environment, commercial breaks are created within content. A set of rules forms a matrix of revenue maximization potential vs. regulatory conditions. Today, this generates workflow within DAM systems by making it necessary for programs to be edited into a certain number of segments. For example, running times may need to be set in order to meet advertising minute regulations. Once in place, then there becomes a whole series of rules that need to be obeyed to connect seller and buyer and monitor the transactions.

In a subscriber environment, where a DAM solution can pump content in either near-videoon-demand (NVOD) or video-on-demand (VOD) form to a consumer, knowledge of that consumer becomes a prerequisite to optimizing the value of the content. Allowing a subscriber to "window-shop" in a virtual manner will generate revenue. However, prompting the consumer with specifically-aimed messages, based upon knowledge of what they buy will inevitably generate more.

For example, how many people using Amazon.com to buy books have been prompted with a message "and other people buying this book have purchased ...?” In some ways it is crude use of information, but it supports the notion that content needs to be sold rather than just published. To achieve this a whole subset of metadata is required to be held, with rules applied to it that deliver recommendations on how content can best be sold to individual consumers.

In an increasingly narrowcast environment, 
where the consumer elects to choose content rather than surf broadcast channels, a genuine value proposition is available to advertisers to reach a desired consumer group more efficiently than any broadcast model. However, delivery requires the availability of extensive consumer information, marrying detailed viewing habits with audience modeling.

In addition, the commercial activity needs to be well managed. Whether this is in the form of standard advertising, 30-second commercials, internet banner, program or content sponsorship, embedded advertising messages or secondary events laid over the top of the primary event, the process needs to be defined and rules applied. Relationships between agency, advertiser, and brand also all need to be captured along with a mechanism to utilize the business intelligence gained earlier to derive a chargeable value. Commissions, sales responsibility, preemption and all the other functions associated with sales activity need to be taken into account, leading finally to an invoicing and as-run procedure. Finally, all events need to be sequenced, transmitted and reconciled.

The business rules become even more complex within a true multimedia operation. Management of TV bookings compared with internet bookings is fundamentally different. One is based on date time, the other being pagebased. Both, however, utilize "impressions" as a measure to allocate a value, and both can be judged against a cost per thousand.

These business rules comprise many man-years of development and therefore investment by software vendors operating in this market. Given that this is a large investment, are there any opportunities that a new application development entails, without going through a full investment cycle?

\section{IMMEDIATE OPPORTUNITIES}

As mentioned earlier vendors are increasingly designing their applications to conform to "open" standards. This generally allows an organization two options in understanding their applications. Over the years, software suppliers have published information of the database structures that underpin their applications, especially the relationships between specific tables. To a degree this allows for accurate collection of data from a wide range of databases but provides little application intelligence. The increasing adoption of a services-oriented architecture and XML protocol, where the properties of a set of code are essentially published, allows a third-party application to utilize functionality much more efficiently.

In theory this would allow a media management system, where business rules such as content acquisition and associated rights usage reside, to expose necessary information to a DAM system. Likewise, a DAM system may be able to show workflow status regarding a piece of content, which, when married to a schedule, date and time from the media management system, could aid prioritization of resources to complete the work associated with a particular event.

With the increasing move towards multimedia environments, it is particularly important to make the visibility of the properties of content and their relationship to distribution more transparent. Although content can be repurposed, content designated for internet usage may not be in an appropriate form for broadcast.

In fact, some organizations are already exploring opening business rules and content properties in this way, either natively between two separate applications or using third-party middleware to facilitate connection and translation of information rules. Initial steps have been tentative, but there are clear opportunities to open up functionality from independent applications to integrate more tightly media management, asset management and devices control to bring broad workflow benefits to an organization.

\section{IN THE LONGER TERM}

Even with services oriented architecture sitting across all component applications, there are limitations to what can be accomplished given the often complex and varied rules acting on content. The increasing digitization of content, delivery and, more importantly, consumer's adoption of personal viewing habits through narrowcast distribution, together with integration of usage information embedded into content through standards such as MPEG-21, mean that we are approaching a catalyst for change.

There was a paradigm shift when paper 
moved to data objects for computers to manage. Similarly, the next evolution may well place all the business rules we currently understand, together with new ones from the developing use of content on top of a DAM application. For the first time the phrase "digital asset management" will truly represent what the application represents to a multimedia operator.

\section{CONCLUSION}

All media organizations understand that the value of content is largely in the data surrounding it and embedded within it. As the data-set increases, so does the content's value. An ability to utilize the data adds greater value and the application of business rules to content delivers this value. 